ISSN 0103-5150

Fisioter. Mov., Curitiba, v. 25, n. 1, p. 55-65, jan./mar. 2012 Licenciado sob uma Licença Creative Commons

\title{
A prevalência de incontinência urinária em mulheres praticantes de jump
}

The prevalence of urinary incontinence in women practicing of jump

\section{Priscilla Pereira de Almeida ${ }^{[a]}$, Lívia Raquel Gomes Machado ${ }^{[b]}$}

[a] Fisioterapeuta especialista em Terapia Manual e Postural pela CESUMAR e em Docência em Ensino Superior pela FAP. Professora do curso de Fisioterapia da Faculdade de Ciências Médicas da Universidade Estadual do Piauí (UESPI), Teresina, PI - Brasil, email: almeidapp@gmail.com

[b] Acadêmica do $10^{\circ}$ período do curso de Fisioterapia da Faculdade de Ciências Médicas da Universidade Estadual do Piauí (UESPI), Teresina, PI - Brasil, email: livianutrifisio@hotmail.com

\section{Resumo}

Introdução: A incontinência urinária (IU) é definida pela Sociedade Internacional de Continência (ICS) como qualquer perda involuntária de urina. Ela exerce múltiplos efeitos sobre as atividades diárias, interações sociais e percepção da saúde. A literatura afirma que a prática de atividades físicas de alto impacto e esforço pode comportar-se como um fator de risco para a IU. Objetivos: identificar a prevalência de IU em um grupo de mulheres praticantes de jump. Materiais e métodos: Estudo transversal quantitativo descritivo, com amostra de 32 voluntárias que responderam a questionário sobre antecedentes obstétricos e prática da atividade física e à tradução do International Consultation on Incontinence Questionnaire - Short Form (ICIQ-SF). Resultados: Houve relato de perdas urinárias em 37,5\% das entrevistadas. A média de idade das mulheres que referem incontinência foi de 31,58 anos (DP $\pm 9,39$ ). As mulheres com filhos tiveram 1,2 mais chances de apresentar IU do que as nulíparas. As que fazem jump três ou mais vezes por semana apresentaram 2,45 mais chances de apresentar IU. Nas que sentiam vontade de urinar durante o jump, a chance de IU foi seis vezes maior. 62,5\% das entrevistadas referiram nenhum impacto na qualidade de vida, e a atividade física foi apontada como situação mais comum de IU. Conclusão: Existe incontinência urinária nas 
praticantes de jump investigadas, multíparas e nulíparas, em jovens, durante a prática de jump e em outras ocasiões, apontando a necessidade de estudos mais abrangentes sobre a temática.

Palavras-chave: Soalho pélvico. Incontinência urinária. Mulheres. Exercício.

\section{Abstract}

Introdution: The urinary incontinence (UI) is defined by International Continence Society (ICS) as any involuntary lost of urine. It exerces numerous effects about the diary activities, social interaction and the perception of health. The literary affirms that the practice of physical activities of high impact and effort, can behave like a risk factor for the UI. Objectives: Identify the prevalence of UI in a group of women practicing of jump. Materials and methods: A descriptive cross-sectional quantitative study, with a sample of 32 volunteers that answered the questionnaire about obstetric records and practice of physical activities and traduction of International Consultation on Incontinence Questionnaire - Short Form (ICIQ-SF). Results: There was urinary lost in $37,5 \%$ of the interviewee. The medium age of the incontinents was about 31,58 years (DP $\pm 9,39$ ). The women with sons had 1,2 more chances of shows UI than the nuliparous. Those who practice jump three or more times in a week, slows 2,45 more chances of shows UI. Those who want to urinate during the jump, the chance of UI was 6 times more. 62,5\% of the incontinents has none impact in quality of life, and practice of physical activities was pointed as situation most common of UI. Conclusion: There is urinary incontinence in practitioners of jump investigated, multiparous and nuliparous, in youngs, during the practice of jump and in other occasions, pointing the necessity of study more comprehensive about the theme.

Keywords: Pelvic floor. Urinary incontinence. Women. Exercise.

\section{Introdução}

A incontinência urinária (IU) é definida pela Sociedade Internacional de Continência (ICS) como qualquer perda involuntária de urina (1). É uma patologia que exerce múltiplos efeitos sobre as atividades diárias, interação social e percepção da própria saúde, principalmente relacionados ao bem-estar social e mental, incluindo problemas sexuais, isolamento social, baixa autoestima e depressão, afetando de modo significativo a qualidade de vida (2). É vista como um problema que afeta mulheres multíparas mais velhas, embora existam evidências de que, durante atividades físicas estressantes, seja comum entre mulheres jovens, fisicamente ativas, mesmo na ausência de fatores de risco conhecidos (3).

A continência é decorrente de um equilíbrio entre as forças de expulsão e as de retenção. Ela decorre, ainda, da posição intrapélvica da uretra proximal, em relação ao colo vesical, onde o aumento da pressão intra-abdominal, quando presente, deve ser transmitido diretamente à uretra. Um assoalho pélvico hipotônico impede a transmissão ideal dessa pressão, a qual não se propagará, pontualmente, até a junção uretrovesical e propiciará a perda urinária $(4,5)$.
Tendo em vista que a incontinência urinária é uma patologia incômoda e vergonhosa - vista erroneamente como uma doença senil -, em mulheres jovens tal condição é ainda mais estigmatizada, pouco difundida e esclarecida, sendo que essas mulheres comumente não se sentem a vontade para recorrer aos profissionais de saúde e relatar a situação.

As literaturas pesquisadas colocam o exercício físico de alto impacto como um fator de risco para a incontinência urinária, mas não são relatadas referências específicas acerca da modalidade jump. Este, que consiste em um programa de exercícios ritmados sobre um minitrampolim, tem se difundido nas academias e se tornado uma das atividades mais requisitadas pelas mulheres, em razão do prazer e motivação que proporciona, além da obtenção ou manutenção dos níveis adequados de condicionamento físico por meio do aumento do gasto energético, da massa corporal magra, do dispêndio de energia pós-exercício e da redução do perfil lipídico, de uma forma dinâmica e divertida (6).

Diante do relato na literatura especializada da elevada prevalência da IU em mulheres, especialmente naquelas que praticam atividades físicas, associado à inexistência de pesquisas que abordem o 
jump e a IU, surgiu a ideia de realizar este estudo, que correlaciona essas duas entidades. Objetivouse, portanto, identificar a prevalência de IU em um grupo de mulheres praticantes de jump, investigando a associação de fatores de risco para IU como idade e paridade a tal exercício físico, além de verificar situações em que há perda urinária nas praticantes, relacionar a frequência da atividade com IU e avaliar o impacto na qualidade de vida provocado pela IU por meio da percepção das incontinentes.

\section{Metodologia}

A pesquisa caracterizou-se como um estudo transversal quantitativo, realizado por meio da análise de uma série de casos em uma amostra de conveniência, no período de setembro a outubro de 2010, da qual participaram mulheres de 18 a 49 anos, residentes no município de Teresina, Piauí, praticantes de jump em três academias, cuja autorização para a realização da pesquisa foi previamente cedida.

As praticantes de jump foram abordadas nas academias ao término das aulas. As voluntárias a participar do estudo foram esclarecidas sobre os objetivos da pesquisa e foi-lhes fornecido o Termo de Consentimento Livre e Esclarecido. Elas responderam um questionário elaborado pelos pesquisadores contendo indagações sobre antecedentes obstétricos e a prática da atividade física (Apêndice A). Acrescido a esse questionário se aplicou a tradução do International Consultation on Incontinence Questionnaire - Short Form (ICIQ-SF) (Anexo A) para a língua portuguesa.

O ICIQ-SF é um questionário simples, breve e autoadministrável, escolhido por avaliar o impacto da IU na qualidade de vida e a qualificação da perda urinária dos pacientes analisados. O ICIQ-SF é composto de quatro questões que avaliam a frequência, a gravidade e o impacto da IU, além de um grupo de oito itens relativos a causas ou situações de IU vivenciadas pelos respondentes. 0 escore geral é obtido pela soma dos escores das questões 3 , 4 e 5. Quanto maior o escore maior o impacto sobre a qualidade de vida (6). 0 impacto sobre a qualidade de vida foi dividido de tal forma: nenhum impacto (0 ponto); impacto leve (de 1 a 3 pontos); impacto moderado (de 4 a 6 pontos); impacto grave (de 7 a 9 pontos); e impacto muito grave (10 ou mais pontos) (7).
Partindo da definição de incontinência urinária da International Continence Society (ICS) como a queixa de qualquer perda involuntária de urina (3), atribuiu-se que seriam consideradas incontinentes as mulheres que respondessem "apresentar perda urinária" no questionário dos pesquisadores ou no ICIQ-SF.

Foram excluídas as que se recusassem a assinar o Termo de Consentimento, apresentassem idade inferior a 18 anos ou superior a 49 anos, além das que porventura apresentaram respostas contraditórias.

Os dados quantitativos foram inseridos em uma planilha eletrônica do programa Excel, versão 2007. Para a análise dos dados foi utilizado o programa estatístico Bioestat 5.0.

Esta pesquisa recebeu parecer favorável do Comitê de Ética em Pesquisa da Faculdade Novafapi, conforme estabelece a Resolução 196/96 do Conselho Nacional de Saúde, pelo processo CAAE n. 0350.0.0453.000-10, em 03 de setembro de 2010.

\section{Resultados}

Participaram desta pesquisa 32 mulheres, a idade média das participantes foi de 29,9 anos (DP \pm $7,1)$. Na questão relacionada ao estado civil, 58,82\% das entrevistadas responderam ser solteiras; 29,41\% declararam-se casadas; 5,88\%, divorciadas; $2,94 \%$, viúvas; e 2,94\% não responderam.

Um percentual de $55,88 \%$ das entrevistadas declarou-se sem filhos. Das que responderam ter filhos, $46,7 \%$ declaram ter um único filho; $26,7 \%$ têm dois filhos; e outras $26,7 \%$ têm três filhos. 0 parto cesáreo foi o mais frequente, ocorrendo em $62,96 \%$, e o parto vaginal ocorreu em 37,03\%.

Não houve relato de perdas urinárias em 62,5\% das entrevistadas. Das que apresentam perdas urinárias, 9,4\% são incontinentes apenas durante o jump; $18,8 \%$ são incontinentes durante o jump e em outras situações; e outras $9,4 \%$ são incontinentes em outras ocasiões, totalizando 37,5\% da amostra (Gráfico 1).

A média de idade das que não relataram perdas urinárias foi de 28,9 anos (DP $\pm 5,65$ anos). Nas que referem perdas de urina, a média de idade foi de 31,58 anos (DP $\pm 9,39$ ) (Tabela 1). Observou-se que todas as mulheres de idade acima dos 40 anos apresentavam perdas urinárias.

Entre as mulheres que não têm filhos (nulíparas), $45 \%$ apresentam queixas de perdas urinárias, 


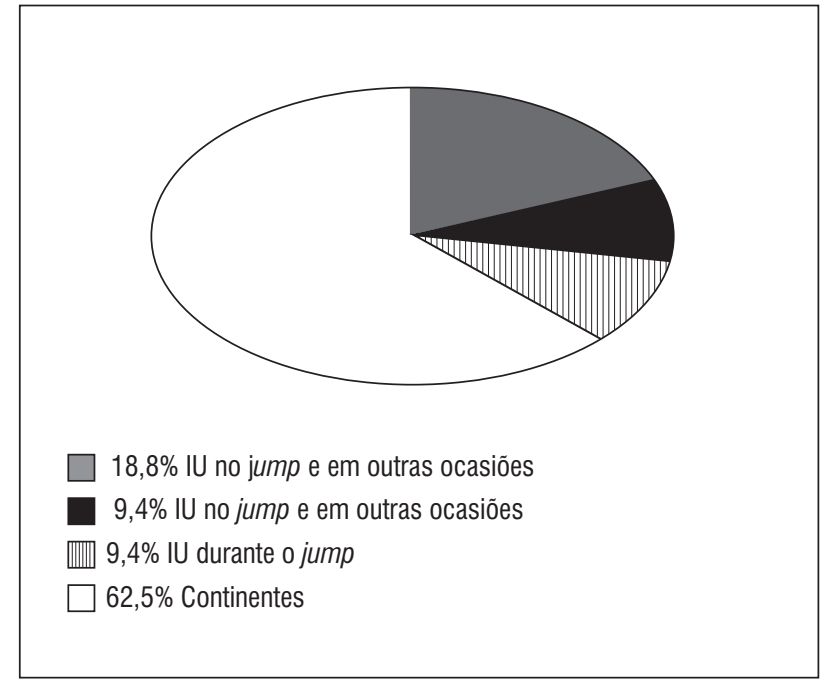

Gráfico 1 - Continência e incontinência urinária em mulheres praticantes de jump

Fonte: Dados da pesquisa.

enquanto dentre as que possuem filhos $50 \%$ relataram ser incontinentes. Observou-se então que as mulheres que têm filhos tiveram 1,2 mais chances de apresentar IU que as nulíparas (Tabela 1).

Em relação aos tipos de parto e IU, das mulheres incontinentes $66,7 \%$ realizaram partos cesáreos; $16,7 \%$ realizaram parto vaginal; e outras $16,7 \%$ realizaram ambos os tipos (Tabela 1).

A Tabela 2 ressalta que a maioria das voluntárias realizava o jump associado a outras atividades $(93,75 \%$ do total), sendo $33,3 \%$ incontinentes.
Todas as que realizavam apenas jump $(6,25 \%$ das entrevistadas) descreveram ser incontinentes.

Quanto à frequência semanal da atividade, observou-se por um cálculo de razão de chances que as mulheres que realizavam a prática três ou mais vezes semanais apresentavam 2,45 mais chances de desenvolver IU (Tabela 2).

Foi observado, por um cálculo de razão de chances, que as mulheres praticantes de jump há menos de 6 meses apresentaram 0,58 mais chances de IU do que as que praticavam há mais de 6 meses.

Indagadas sobre sentir vontade de urinar durante a atividade, $25 \%$ das continentes sentem vontade de urinar. Dentre as incontinentes, $66,7 \%$ sentem vontade de urinar e 33,7\% não sentem. Analisandose esses dados por meio de um cálculo de razão de chances, observa-se, então, que nas mulheres que sentem vontade de urinar a chance de IU foi seis vezes maior (Tabela 2).

Quanto à frequência de perda urinária, 50\% definiram perder uma vez por semana ou menos; $33,3 \%$ relatam perda entre duas a três vezes por semana; e $16,7 \%$ relatam perder urina diversas vezes ao dia. Quando indagadas sobre a quantidade de perda urinária que apresentam, 75\% delas relatam perder uma pequena quantidade e $25 \%$ referem uma moderada perda.

A interferência da IU na vida diária, pelo ICIQ$\mathrm{SF}$, foi quantificada pelas respostas às questões 3,4 e 5 do ICIQ-SF (8), e em $62,5 \%$ das entrevistadas a IU não representava nenhum impacto na qualidade

Tabela 1 - Descrição do perfil obstétrico em mulheres praticantes de jump e continência/IU

\begin{tabular}{lcc}
\hline & Continentes & Incontinentes \\
& $\mathrm{N}(\%)$ & $\mathrm{N}(\%)$ \\
\hline Idade (média em anos) & $28,9(\mathrm{DP} \pm 5,65$ anos) & $31,58(\mathrm{DP} \pm 9,39)^{*}$ \\
\hline Paridade & $11(55 \%)$ & $6(50 \%)$ \\
Sem filhos & $9(45 \%)$ & $6(50 \%)$ \\
Com filhos & & $4(66,7 \%)$ \\
\hline Tipo de parto & $5(55,6 \%)$ & $1(16,7 \%)$ \\
\hline Césareo & $3(33,3 \%)$ & $1(16,7 \%)$ \\
Vaginal & $1(11,1 \%)$ & $(5 \%)$ \\
Ambos & & \\
\hline
\end{tabular}

Fonte: Dados da pesquisa. 
Tabela 2 - Prática da atividade física e continência/IU

\begin{tabular}{lrr}
\hline & Continentes & Incontinentes \\
\hline & $\mathrm{N}(\%)$ & $\mathrm{N}(\%)$ \\
\hline Quanto ao tipo de atividade física & $0(0 \%)$ & $2(6,25 \%)$ \\
\hline Apenas jump & $20(62,5 \%)$ & $10(31,25 \%)$ \\
Jump e outras & & $3(25 \%)$ \\
\hline Quanto à frequência semanal & $9(45 \%)$ & $5(41,6 \%)$ \\
\hline 1-2 vezes semanais & $7(35 \%)$ & $4(33,3 \%)$ \\
3 vezes semanais & $4(20 \%)$ & $3(33,3 \%)$ \\
Mais de 3 vezes semanais & & $3(25 \%)$ \\
\hline Quanto ao tempo de prática & $9(45 \%)$ & $5(41,6 \%)$ \\
\hline Mais de 1 ano & $3(15 \%)$ & $8(25 \%)$ \\
Mais de 6 meses & $8(40 \%)$ & $4(12,5 \%)$ \\
Menos de 6 meses & & \\
\hline Quanto à vontade de urinar durante a atividade & $5(15,6 \%)$ & $6(18,75 \%)$ \\
\hline Sentem & $15(46,8 \%)$ & $6(18,75 \%)$ \\
\hline Não sentem & & $0(0 \%)$ \\
\hline Quanto à perda de urina durante o jump & $20(62,75 \%)$ & \\
\hline Relatam perder & & \\
Não perdem & & \\
\hline
\end{tabular}

Fonte: Dados da pesquisa.

de vida, porém impactos leves a graves puderam ser observados (Gráfico 2).

Quando indagou-se sobre todas as ocasiões em que apresentam perdas urinárias, a situação mais citada foi durante a atividade física, que abrangeu $25 \%$ das entrevistadas. Perdas urinárias antes de chegar ao banheiro ou ausência de razão óbvia notada para a perda urinária também tiveram percentual relevante $(16,7 \%$ cada). Outras situações também foram descritas como ocasiões de perdas urinárias, tais como na tosse/espirro, antes de chegar ao banheiro, após o término de urinar e ao estarem se vestindo, e associações de tosse/espirro com a atividade física e/ou dormindo, conforme descreve o Gráfico 3.

\section{Discussão}

O presente estudo, por meio da análise de uma série de casos, mostra que a incontinência urinária feminina em mulheres praticantes de jump é uma patologia presente, que a prevalência da IU aumentou com a idade e que ocorre também em mulheres jovens e nulíparas. A prevalência da IU aumenta com a idade, com uma taxa típica em jovens adultas de $20 \%-30 \%$, um pico em torno da meia-idade (30\%-40\% de prevalência) e um aumento nas mulheres mais velhas (prevalência de 30\%-50\%) (8, 9). Outros estudos que destacaram a IU em jovens expõem que 7\%-37\% mulheres com idade entre 20 39 anos relatam alguma IU (10); altas prevalências em mulheres com infecções no trato urinário, naquelas que não usam medicação estrogênica, nulíparas e em mulheres com um ou dois filhos, além de maior frequência de IU dentre as que têm filhos quando comparadas a nulíparas. (11).

Nesta investigação, as mulheres que têm filhos tiveram 1,2 mais chances de apresentar IU que as nulíparas. Tal ocorrência é justificada pelo fato de aquelas estarem expostas à gravidez e ao parto e suas implicações (alterações hormonais, peso do 


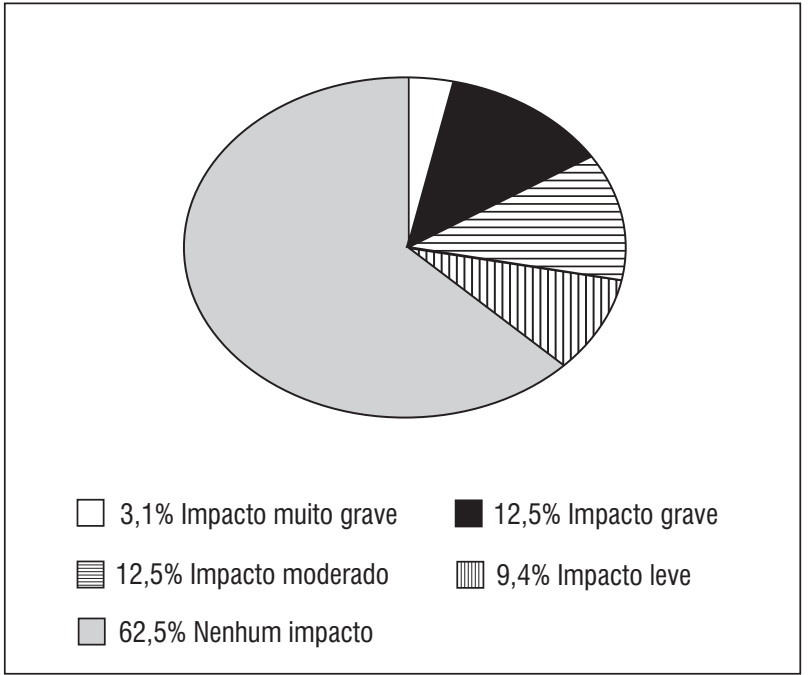

Gráfico 2 - Impacto da IU na qualidade de vida de mulheres praticamentes de jump

Fonte: Dados da pesquisa.

recém-nascido, durações prolongadas no parto vaginal, uso de instrumentação cirúrgica), além do aumento do índice de massa corpórea (IMC) e da diabetes $(9,10,12,13)$. Todavia, nos dados obtidos não se nota significativa presença de IU associada a partos vaginais, uma vez que $66,7 \%$ das pesquisadas incontinentes realizaram partos cesáreos.

Nas nulíparas, a incontinência urinária aos esforços tem como fatores de risco: obesidade central, constipação, dor pélvica crônica, histórico de doenças respiratórias, menopausa, consumo de álcool e cafeína, tabagismo, uso de drogas, doenças crônicas, fatores hereditários e a prática de exercícios físicos (14-16).

A causa para a alta prevalência de IU em jovens e nulíparas não é definida, porém acredita-se que os ligamentos cardinal e uretro-sacral e o tecido conjuntivo podem ser lesados e perder sua eficiência mecânica decorrente dos aumentos repetitivos da pressão abdominal, ocasionados por trabalhos manuais pesados e tosse crônica. Mulheres jovens e nulíparas não apresentam rupturas de ligamentos, lesão de fáscias, lesão das fibras musculares nervosas e do assoalho pélvico causadas por partos e gestações. Sendo assim, a presença de incontinência nessas mulheres pode decorrer de fraqueza genética do tecido conjuntivo, da localização mais baixa do assoalho e o número reduzido de fibras musculares nessa região (17). Em mulheres jovens nulíparas com sintomas de

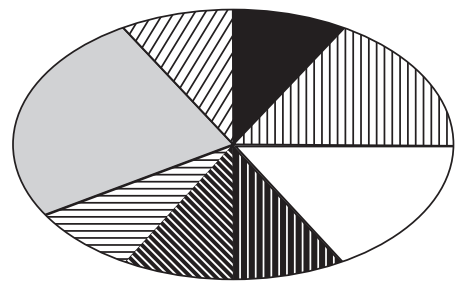

Z $8,3 \%$ Tosse/espirro

8,3\% Abstenção

16,7\% Sem razão óbvia

8,3\% Tosse/espirro e na atividade física

Gráfico 3 - Atividades em que houve relato de perda urinária segundo ICIQ-SF

Fonte: Dados da pesquisa.

IU leve, o exercício extenuante resulta em menor pressão de contração vaginal, uma vez que haveria um comprometimento do suprimento sanguíneo e debilidade de oxigênio para as fibras musculares do tipo I, responsáveis pela manutenção do tônus, indicando fadiga muscular do pavimento pélvico, além disso, existe uma redução do sinal neural após atividade muscular prolongada, reduzindo a eficiência da contração muscular e favorecendo a perda da continência $(18,12)$.

Quando a IU é estudada em mulheres praticantes de exercícios físicos, diversos estudos referem que sua fisiopatologia esteja relacionada às pressões constantes sobre o assoalho pélvico e aumentos abruptos da pressão abdominal que ocorrem durante a prática de atividades físicas. Mesmo em mulheres com os músculos do assoalho pélvico (MAP) fortes, a atividade física árdua levaria ao aumento da pressão abdominal, aliada à sobrecarga, ao estiramento e ao enfraquecimento do assoalho pélvico, acarretando em IU $(19,20)$. Movimentos de alto impacto resultam em força de impacto sobre o assoalho pélvico três a quatro vezes maior que o peso corporal (12), mais especificamente relata-se que a força vertical de reação máxima do solo durante diferentes atividades esportivas é três a quatro vezes o peso do corpo quando corremos, cinco a doze vezes pulando, e nove vezes na queda após um salto em altura (20).

As maiores prevalências de IU são em esportes que envolvem atividades de alto impacto, como: 
ginástica, atletismo, fisiculturismo, saltos, exercícios que exijam contrações abdominais máximas repetitivas, além de esportes com mudança abrupta de movimento $(12,20)$. Na atividade jump, são comuns rotações, oscilações de equilíbrio, apoio monopodal e consequentemente a utilização da musculatura abdominal, gerando condições favoráveis para uma significativa prevalência de IU.

Neste estudo, nas mulheres que realizavam a prática com maior frequência semanal a prevalência de IU era mais frequente. Tais achados condizem com Eliasson et al. (21), que observaram que duração e frequência de treinamento são significativamente associadas com a IU. Contudo, confrontam no quesito tempo de prática da atividade, uma vez que, nessa amostra, as praticantes há menos de 6 meses foram as que mais expuseram apresentar perdas de urina, e tal autor aponta que as perdas urinárias costumam surgir num intervalo de 1-4 anos de treino após início de treino esportivo. Tal fato pode ser atribuído à hipotonicidade do assoalho pélvico nas pesquisadas, a maiores IMC e sobrepeso ou à menor força abdominal (22), uma vez que o pouco tempo de prática de atividade física pode estar associado ainda à existência desses vestígios do sedentarismo.

Indagadas sobre sentir vontade de urinar durante a atividade, dentre as incontinentes, $66,7 \%$ sentem vontade de urinar e 33,7\% não sentem, porém $50 \%$ delas perdem urina. Observa-se, então, que nas que sentem vontade de urinar a chance de IU foi seis vezes maior. A literatura reporta pesquisas com resultados de perda urinária involuntária durante o treino em $80 \%$ das atletas acompanhadas (21).

Com relação à intensidade da perda de urina, os fatores envolvidos no seu determinismo que mais causam impacto negativo na qualidade de vida são as queixas de dois ou mais episódios de perda de urina por dia, perda em jato, ou necessidade de mais de quatro absorventes por dia (23). Na amostra estudada, apenas 16,7\% (duas mulheres) relatam perder urina diversas vezes ao dia e, considerando a quantidade de urina, $75 \%$ da amostra descreve perder uma pequena quantidade.

Considerando o impacto na qualidade de vida, $62,5 \%$ das entrevistadas referia que a IU representava nenhum impacto; 9,4\% consideravam um impacto leve; $12,5 \%$ impacto moderado; $12,5 \%$ impacto grave; e 3,1\% impacto muito grave. Esses resultados foram semelhantes aos encontrados em estudo brasileiro com 875 mulheres de idade média de 47,6 anos que considerou o impacto da IU na qualidade de vida das participantes, por meio do questionário ICIQ-SF, constatando que 83,9\% relataram nenhum impacto; $5,2 \%$, impacto leve; $4,1 \%$, impacto moderado; $3,6 \%$ mostraram impacto grave; e 3,2\%, muito grave (24).

Tal evento pode ser relacionado ao fato da amostra consistir em mulheres jovens, considerando estudos prévios que descrevem que os relatos de IU severa aumentam com a idade, sendo em média $8 \%$ das mulheres com idade de 30 a 39 anos e $13 \%$ entre as de 40 a 49 anos que descrevem IU grave (9). A incontinência urinária, com seus sintomas associados, pode causar significativo impacto na qualidade de vida e considerável variedade de percepções e respostas entre as pessoas (25). As mulheres costumam não dar relevância à IU, por vergonha quando jovens e resignação quando mais velhas (26), uma vez que o controle das eliminações é construído socialmente, assim como os estigmas em torno da IU (27). Por outro lado, há registro de incontinentes que não relatam impacto negativo no bem-estar ou restrição das atividades sociais (28), tal como foi narrado nos achados da amostra pesquisada.

Em estudo australiano, houve uma associação transversal entre perda de urina e atividade física, de tal forma que as mulheres com perdas urinárias mais frequentes também foram mais propensas a relatar baixos níveis de atividade física (29). $\mathrm{Na}$ amostra investigada, a presença de distúrbios urinários não parece ser uma barreira durante o esporte ou exercício, uma vez que a maioria das voluntárias afirmou que a perda urinária não as incomodava pronunciadamente.

0 presente estudo encontrou perdas urinárias descritas nas mais diversas situações, além das perdas na atividade física, anteriormente explicadas, foram encontrados relatos de perdas durante tosse e espirro, em que há aumento da pressão intra-abdominal, e manobra de Valsalva, além de perdas associadas em tais atividades em quase $50 \%$ das pesquisadas, o que sugere necessidade de investigação de IU. Estudos anteriores, assim como observado nesta pesquisa, evidenciam que as perdas aos esforços são as mais relatadas, abrangendo de $50 \%$ a $83 \%$ das mulheres incontinentes $(15,30)$. Houve ainda relato de incontinência associada às atividades fisicas, na tosse/espirro e dormindo. Foram descritas perdas antes de chegar ao banheiro em $16,7 \%$ das 
pesquisadas, e outras 16,7\% não souberam atribuir uma razão óbvia à incontinência; 8,3\% delas descreveram perder urina ao terminar de urinar e estar se vestindo, o que pode ser preditivo de retenção urinária. Em tal investigação não se definiu os tipos de incontinência urinária encontrados, uma vez que se considera a necessidade de uma investigação clínica, além de complementação diagnóstica que auxilie na detecção e quantificação da perda urinária (31), como métodos mais fidedignos para classificação.

\section{Conclusão}

Embora o tamanho da amostra seja de um grupo limitado de mulheres, os achados estão consonantes com a literatura, em que mulheres praticantes de atividade física de impacto relatam perdas urinárias, mesmo na ausência de outros fatores de risco. Todavia, não se pode afirmar que haja prevalências pronunciadas de incontinência urinária em mulheres praticantes de jump.

Apesar de este estudo apresentar limitações, como a utilização de um questionário geral para avaliar o impacto da IU na vida diária, os resultados revelam que, de certa forma, na amostra analisada existe perda urinária considerável. Contudo, reconhece-se a necessidade de um estudo mais apurado, com aplicação de um questionário específico para a qualidade de vida propriamente dita, que considere implicações nos diversos aspectos da vida diária das mulheres incontinentes.

Entretanto, esses resultados são importantes na reformulação da compreensão da IU feminina nas atividades físicas cotidianas, de forma a despertar interesse em abordar essa população. Entretando, há necessidade da realização de estudos mais abrangentes sobre a temática, com amostras maiores, de forma a traçar melhor perfil da problemática e desenvolver estratégias de prevenção e tratamento.

\section{Referências}

1. Abrams P, Cardoso L, Fall M, Griffiths D, Rosier P, Ulmesten $\mathrm{U}$, et al. The standardisation sub-committee of the International Continence Society. Urology. 2003;61(1):37-49.
2. Dedicação AC, Haddad M, Saldanha MES, Driusso P. Comparação da qualidade de vida nos diferentes tipos de incontinência urinária feminina. Rev Bras Fisioter. 2009;13(2):116-22.

3. Lopes MHBM, Higa R. Restrições causadas pela incontinência urinária à vida da mulher. Rev. Esc Enferm USP. 2006;40(1):34-41.

4. Grosse D, Sengler J. Reeducação perineal: concepção, realização e transcrição em prática liberal e hospitalar. São Paulo: Manole; 2002.

5. Moreira SFS, Girão MJBC, Sartori MGF, Baracat EC, Lima GR. Mobilidade do colo vesical e avaliação funcional do assoalho pélvico em mulheres continentes e com incontinência urinária de esforço consoante o estado hormonal. Rev Bras Ginecol Obstet. 2002; 24(6):365-70.

6. Grossl T, Guglielmo LGA, Carminatti L, Silva J. Determinação da intensidade da aula de power jump por meio da freqüência cardíaca. Rev Bras Cineantropom. Desempenho Hum. 2008;10(2):129-31.

7. Ricceto C, Palma P, Tarazona A. Aplicaciones clínicas de la teoría integral de la continencia. Actas Urol Esp. 2005;29(1):31-40.

8. Hunskaar S, Lose G, Sykes D, Voss S. The prevalence of urinary incontinence in women in four European countries. BJU Int. 2004;93(3):324-30.

9. Melville JL, Katon W, Delaney K, Newton K. Urinary incontinence in US women a population-based study. Arch Intern Med. 2005;165(14):538-42.

10. Buckley BS, Lapitan MC. Prevalence of urinary incontinence in men, women, and children-current evidence: findings of the Fourth International Consultation on Incontinence. Urology. 2010;76(2):265-71.

11. Hägglund D, Olsson H, Leppert J. Urinary incontinence: an unexpected large problem young females. Results from a population-based study. Fam Pract. 1999;16(5):506-9.

12. Moreno AL. Fisioterapia em uroginecologia. 2a ed. Barueri: Manole; 2009.

13. Oliveira SG, Battisti BZ, Secco VL. Avaliação da qualidade de vida de portadores de incontinência urinária. RBCEH. 2009;6(1):34-41. 
14. Contreras Ortiz O. Stress urinary incontinence in the gynecological practice. Int J Gynaecol Obstet. 2004; 86(Suppl 1):S6-16.

15. Zhu L, Lang C, J Liu, S Han, J Huang, Li X. The epidemiological study of women with urinary incontinence and risk factors for stress urinary incontinence in China. Menopause. 2009;16(4):831-6.

16. Caetano AS, Tavares MCGC, Lopes MHBM. Incontinência urinária e a prática de atividades físicas. Rev Bras Med Esporte. 2007;13(4):270-4.

17. Santos ES, Caetano AS, Tavares MCGCF, Lopes MHBM. Incontinência urinária entre estudantes de educação física. Rev Esc Enferm USP. 2009;43(2):307-12.

18. Ree ML, Nygaard I, Bø K. Muscular fatigue in the pelvic floor muscles after strenuous physical activity. Acta Obstet Gynecol Scand. 2007;86(7):870-6.

19. Fozzatti MCM, Palma P, Herrmann V, Dambros M. Impacto da reeducação postural global no tratamento da incontinência urinária de esforço feminina. Rev Assoc Med Bras. 2008;54(1):17-22.

20. Araújo MP, Oliveira E, Zucchi EVM, Trevisani VFM, Girão MJBC, Sartori MGF. Relação entre incontinência urinária em mulheres atletas corredoras de longa distância e distúrbio alimentar. Rev Assoc Med Bras. 2008;54(2):146-49.

21. Eliasson K, Larsson T, Mattsson E. Prevalence of stress incontinence in nulliparous elite trampolinists. Scand J Med Sci Sports. 2002;12(2):106-10.

22. Moraes NM, Silva DTG. Estudo comparativo da força muscular do assoalho pélvico em mulheres sedentárias e mulheres que praticam atividade física. [dissertação]. Belém: Universidade da Amazônia; 2006.

23. Tamanini JTM, D’ancona CAL, Botega NJ, Netto NR, Jr. "Validação do King's Health Questionnaire" para o português em mulheres com incontinência urinária. Rev Saúde Pública. 2003;2(37):203-11.

24. Oliveira JMS. Prevalência da incontinência urinária e sua associação com a obesidade em mulheres na transição menopausal e após menopausa. [dissertação]. São Paulo: Universidade de São Paulo; 2010.
25. Klüber L, Moriguchi EH, Cruz IBM. A influência da fisioterapia na qualidade de vida de mulheres com incontinência urinária: revisão. Rev Med PUCRS. 2002; 12(3):243-49.

26. Hirai K, Sumi T, Kanaoka Y, Ishiko O. Female urinary incontinence: diagnosis, treatment and patients' concerns. Drugs Today (Barc). 2002;38(7):487-93.

27. Borba AMC, Lelis MAS, Brêtas ACP. Significado de ter incontinência urinária e ser incontinente na visão das mulheres. Texto \& Contexto Enferm. 2008; 17(3):527-35.

28. Abreu NS. Qualidade de vida na perspectiva de idosas submetidas à fisioterapia para incontinência urinária. [dissertação]. Belo Horizonte: Universidade Federal de Minas Gerais; 2006.

29. WJ Brown, Miller YD. Too wet to exercise? Leaking urine as a barrier to physical activity in women. J Sci Med Sport. 2001;4(4):373-8.

30. Siracusano S, Pregazzi R, D'Aloia G, Sartore A, Di Benedetto P, Pecorari V, et al. Prevalence of urinary incontinence in young and middle-aged women in an Italian urban area. Eur J Obstet Gynecol Reprod Biol. 2003;107(2):201-4.

31. Nascimento SM. Avaliação fisioterapêutica da força muscular do assoalho pélvico na mulher com incontinência urinária de esforço após cirurgia de Wertheim-Meigs: revisão de literatura. Rev Bras Cancerol. 2009;55(2):157-63.

Recebido: $15 / 08 / 2010$

Received: 08/15/2010

Aprovado: 28/04/2011

Approved: 04/28/2011 


\section{Apêndice}

\section{Questionário de identificação pessoal e histórico ginecológico}

Iniciais

Data de nascimento

Idade

Estado civil

Histórico ginecológico

1. Tem filhos? ( ) sim ( ) não quantos?

2. Quais os tipos de parto?

( ) normal quantos? ( ) cesariana quantos?

3. Realizou episiotomia? ( ) sim ( ) não

4. Realizou alguma cirurgia ginecológica? ( ) sim ( ) não qual?

Atividade física

Quais atividades físicas você realiza?

( ) apenas jump ( ) musculação e jump ( ) jump e step ( ) outras

Qual a frequência que você realiza a atividade jump?

( ) 1 a 2 vezes por semana ( ) 3 vezes por semana ( ) mais de 3 vezes por semana

Há quanto tempo pratica atividade?

( ) mais de um ano ( ) mais de 6 meses ( ) menos de 6 meses

Por que você escolheu o jump?

( ) pelo alto gasto energético ( ) pela dinamicidade ( ) pela diversão ( ) outros

* Durante o jump

1) Sente vontade de urinar?

( ) sim ( ) não

2) Você perde urina?

( ) $\operatorname{sim}$ ( ) não 


\section{Anexos}

\section{ICIQ-SF}

Muitas pessoas perdem urina algumas vezes. Estamos tentando descobrir quantas pessoas perdem urina e o quanto isso as aborrece. Ficaríamos agradecidos se você pudesse nos responder as seguintes perguntas pensando como você tem passado em média nas últimas quatro semanas.

1) Data de nascimento:__/____

2) Sexo: masculino ( ) feminino ( )

3) Com que freqüência você perde urina? (assinale uma resposta)

0 nunca ( )

1 uma vez por semana ou menos ( )

2 duas ou três vezes por semana ( )

3 uma vez ao dia ( )

4 diversas vezes ao dia ( )

5 o tempo todo ( )

4) Gostaríamos de saber a quantidade que você pensa que perde.

Nenhuma ( ) 0

Uma pequena quantidade ( ) 2

Uma moderada quantidade ( ) 4

Uma grande quantidade ( ) 6

5) Em geral quanto que perder urina interfere em sua vida diária? Por favor, circule um numero entre 0 (não interfere) e 10 (interfere muito).

\begin{tabular}{lcrllllllrr}
0 & 1 & 2 & 3 & 4 & 5 & 6 & 7 & 8 & 9 & 10 \\
Não interfere & & & & & \multicolumn{7}{rl}{ interfere muito }
\end{tabular}

ICIQ-SCORE: soma dos resultados $3+4+5=$

6) Quando você perde urina? Por favor assinale todas as alternativas que se aplicam a você.

nunca ( )

perco antes de chegar ao banheiro ( )

perco quando estou dormindo ( )

perco quando tusso/espirro ( )

perco quando estou fazendo atividade física ( )

perco quando terminei de urinar e estou me vestindo ( )

perco sem razão óbvia ( )

perco o tempo todo ( ) 